\title{
Optimización de cobertura para lugares georreferenciados
}

\section{Optimization of coverage for georeferenced zones}

\author{
Edison Marcelo Rodríguez-Herrera \\ Ingeniera Eléctrica (c) \\ Universidad Politécnica Salesiana \\ Quito, Ecuador \\ erodriguezh@est.ups.edu.ec
}

\author{
Marjorie Alexandra Angamarca-Guamán \\ Ingeniera Eléctrica (c) \\ Universidad Politécnica Salesiana \\ Quito, Ecuador \\ mangamarcag@est.ups.edu.ec
}

\author{
Esteban Mauricio Inga-Ortega \\ Ph. D. (c) en Ingeniería \\ Universidad Politécnica Salesiana \\ Quito, Ecuador \\ einga@ups.edu.ec
}

\begin{abstract}
Resumen- En este artículo se presenta un análisis de las redes alámbricas e inalámbricas en los métodos tradicionalmente utilizados al aire libre, consideraremos la optimización en función de la capacidad, la cobertura e interferencia de un escenario georreferenciado; nos basaremos en el uso de algoritmos que nos ayudarán a la simulación de la conexión más óptima, el cual nos permitirá tener un nuevo análisis del escenario y ser capaz de ahorrar en los presupuestos de los equipos, conexiones y tiempo de instalación, así podemos garantizar la cobertura total para los usuarios. El cual el instalador tendrá la posibilidad de ver la conexión más adecuada para su escenario. El resultado del algoritmo presentado en una gráfica se podrá visualizar tanto la capacidad como la cobertura de la red de comunicación. Empezaremos con una cobertura mínima hasta llegar a la cobertura total de todos los usuarios.
\end{abstract}

Palabras clave- Capacidad, cobertura, kruskal, optimización, Outdoor, Prim.

Abstract- This article presents an analysis of the wired and wireless networks in the methods traditionally used outdoors, we will consider the optimization based on the capacity, coverage and interference of a georeferenced scenario; we will be based on the use of algorithms that will help us to simulate the optimal connection, which will allow us to have a new analysis of the scenario and be able to save on equipment budgets, connections and installation time, so we can guarantee the total coverage for users. The installer will have the possibility to see the most suitable connection for your scenario. The result of the algorithm presented in a graph will be able to visualize both the capacity and the coverage of the communication network. We will begin with a minimum coverage until we reach the total coverage of all users.

Keywords- Capacity, coverage, kruskal, optimization, Outdoor, Prim.

\section{INTRODUCCIÓN}

En la infraestructura de medición avanzada (AMI) etapa fundamental dentro de una red eléctrica inteligente, se requiere de una red de comunicaciones robusta y madura que permita cubrir en primer lugar la demanda de clientes del sistema eléctrico, los mismos que deben ser analizados en la planificación AMI y en su diseño también [1].

Este trabajo se centra en el proceso de distribución de ancho de banda con el fin de proporcionar una asignación de recursos equitativa y eficaz cognitivo. Al optimizar el proceso de asignación de ancho de banda en las redes FIWI, el rendimiento de la entrega de datos se mejora de manera espectacular, lo que permite a más usuarios su servicio, y altos estándares de calidad de servicio (QoS) que deben abordarse [2].

Diferentes algoritmos de grafos se utilizan ampliamente en el diseño de red y el problema de aná- 
lisis. El problema de árbol de expansión mínima de restricción de grado se utiliza en el diseño de redes de telecomunicaciones, diseño de circuitos integrados y la planificación hoja de ruta [3].

La red FIWI reúne las ventajas de la red de acceso óptica y de acceso inalámbrico. Para las nuevas redes debe existir compatibilidad con servicios actuales, con un soporte de múltiples servicios, estas redes deben tener fiabilidad y calidad de servicio. Estas redes inalámbricas pueden ser clasificadas de diferentes campos, como ser basada en cobertura y tipo de información como son voz y dato. Para el desarrollo de la red inalámbrica se analizara la cobertura, el enrutamiento e interferencia, donde las cuales puedan existir en la red. [4].

La aplicación de técnicas de optimización para los problemas de alimentación y parece que hay una competencia entre la sofisticación de los algoritmos de optimización y la creciente complejidad de la planificación y las operaciones relacionadas con los problemas del sistema eléctrico [5].

Con el rápido aumento en la demanda de acceso inalámbrico de banda ancha, la industria WLAN ha sido desarrollada recientemente. Sin embargo, la optimización de la cobertura sigue siendo un serio desafío para la implementación de WLAN. Una gran cantidad de investigación se ha llevado a cabo en la optimización de la cobertura de WLAN [6].

Las redes inalámbricas se caracterizan por una política de asignación de canal / espectro fijo. Para la asignación optima de agujeros de espectro disponibles a los recursos y hay un aumento exponencial en el acceso al espectro limitado de servicios móviles en los últimos años [7]

Se espera que las redes de sensores tengan un impacto significativo en una amplia gama de aplicaciones que va desde lo militar a lo científico, también se lo puede encontrar en la industria ,en el área de la salud y el medio ambiente, el establecimiento de redes de sensores inalámbricos ubicuos que impregnará la sociedad [8].

\section{ANÁLISIS DE COMUNICACIONES}

\subsection{Despliegue de cobertura de comunicaciones inalámbricas}

LAN (WLAN) se están volviendo cada vez más populares para proporcionar acceso a la red de alta velocidad de datos para equipos móviles. A diferencia de los sistemas tradicionales de telefonía celular, WLAN está desplegada de manera ad hoc, a menudo basada en una conjetura por la persona que instale los puntos de acceso (APs). Normalmente, esto se traduce en brechas de cobertura o pérdida de capacidad, debido a los puntos de acceso fuera de lugar. En este trabajo, se explora el uso de algoritmos heurísticos sencillos, tales como la poda y la zona de búsqueda para obtener una solución óptima al problema de emplazamiento AP [9].

La tecnología WLAN ha tenido una sorprendente difusión en el mercado de las telecomunicaciones. WLAN puede verse como extensión de la cobertura máxima. Se han desarrollado heurísticas eficaces capaces de proporcionar soluciones casi óptimas para una cantidad razonable de tiempo. [10].

Una WLAN está básicamente constituida por uno o más Puntos de Acceso inalámbricos (AP) conectados a la red troncal que proporciona conexión inalámbrica al espacio abierto [11].

La arquitectura de una WMN al outdoor, en la cual es una celda de malla pero esta celda se dice que cada usuario envía o recibe el tráfico para otros al punto central de puerta de enlace / acceso (AP) [12].

Con las ventajas de una implementación rápida y un costo menor, la red de salto múltiple es una tecnología de prometedora difusión de la información para la aplicación de las redes de acceso outdoor. La implementación amplia del salto múltiple da un despliegue de redes públicas al aire libre y se ha convertido en un tema crítico. Tradicionalmente, la implementación de redes outdoor es un reto porque la conexión de los puntos de acceso (APs) a través de cables necesita una gran cantidad de trabajos de cableado costoso pero con el 
salto múltiple es una solución económica el cual admite el acceso de banda ancha ubicado en la área externa [13].

\subsection{Despliegue de cobertura de comunicaciones alámbricas}

La red de fibra Wireless (Fiwi), que tiene un prometedor futuro, es una combinación óptima de una subred de fibra hacia atrás y una subred inalámbrica hacia adelante. En la subred de fibra de la red Fiwi, el terminal de línea óptica (OLT) se coloca en la oficina central (CO) y conectado a través de la fibra a varias unidades de red óptica (unidades ONU) [14].

El objetivo final de la Internet y la comunicación de redes en general es proporcionar el acceso a información, cuando lo necesitamos. En la actualidad es muy claro que los usuarios deben tener un servicio excelente de banda ancha, redes de acceso de banda ancha futuras deben aprovechar en ambas tecnologías y ellos convergen a la perfección, dando lugar a fibras de acceso inalámbrico (FIWI) [15].

En los próximos años se espera que las redes de acceso de banda ancha tengan algunos cambios. En primer lugar, el cableado hecho de cobre va a tener un papel menos importante así dando paso a las redes de acceso de fibra bimodal-inalámbrico (FIWI). En segundo lugar, las redes de acceso de banda ancha sé volverán menos perjudiciales al ambiente [16].

Tenemos como análisis terminal de línea óptica (OLT) en la oficina central (CO) y se conecta un divisor a través de la fibra de alimentación con terminales de fibras de distribución [17].

Una red FiWi permite la infraestructura de forma global, el cual nos permite el intercambio de información entre las redes y mediante la optimización de puntos de acceso se reducirá la cantidad de equipos así mismo su costo de este. La red FIWI nos permite tener una evolución considerable y basándonos en un esquema de crecimiento a través del tiempo [1].

\section{FORMULACIÓN DEL PROBLEMA}

\subsection{Cobertura de comunicaciones inalámbricas}

Suponemos una red inalámbrica MAN-WAN para una residencial. Cada Estación Base está establecida con muchos canales para dar cobertura a los usuarios. Para el análisis de capacidad y cobertura en el mapa de un barrio para esto nos ayudaremos con la herramienta Matlab.

Nuestro trabajo se centra en dos modelos (LPsolve) con un número $\mathrm{N}$ de usuarios y $\mathrm{M}$ la ubicación posible de Estaciones Base.

Tendremos en consideración la capacidad de los EB, también cuenta el porcentaje de cobertura en la cual queremos cubrir siempre comenzando con un mínimo del $70 \%$ hasta alcanzar la cobertura de todos los usuarios en un porcentaje $X \%$, con un radio de cobertura: 15 , Capacidad: 10, Usuarios: 57, Estaciones Base: 37.

TABLA I

VARIABLES- MODELO DE OPTIMIZACIÓN

\begin{tabular}{|l|c|c|}
\hline Variable de decisión & Simbología & Interpretación \\
\hline Conjunto de usuarios & $\mathrm{N}$ & Número de usuarios \\
\hline Ubicación de A.P. & $\mathrm{Y}$ & A.P. Posibles \\
\hline Ubicación de A.P. & $\mathrm{Z}$ & A.P. Activos \\
\hline Conjunto de A.P & $\mathrm{M}$ & Números de A.P. \\
\hline Cobertura & $\mathrm{P}$ & $\begin{array}{c}\text { Probabilidad de } \\
\text { cobertura (\%) }\end{array}$ \\
\hline Alcance de A.P. & $\mathrm{R}$ & Radio de cobertura \\
\hline Capacidad de A.P. & $\mathrm{C}$ & $\begin{array}{c}\text { Número de usua- } \\
\text { rios conectados }\end{array}$ \\
\hline
\end{tabular}

Fuente: los autores

En la Tabla I podemos observar cada elemento utilizado en el seudocódigo para el modelo de optimización.

La función objetivo: es expresada de la siguiente manera, (1) - (5)

$$
\min \left(\sum_{i=1}^{M} Z_{i}\right)
$$


Sujeto a:

$$
\begin{gathered}
Y_{j}=\sum_{i=1}^{M} X_{i, j} ; \Lambda_{j} \varepsilon U \\
\sum_{j=1}^{N}\left(X_{i, j} \leq C Z_{i}\right) ; \Lambda_{j} \varepsilon S \\
\sum_{j=1}^{N}\left(Y_{i} \geq N \cdot \text { Porc }\right) \\
X_{i, j} \leq \alpha_{i, j} Z_{i} ; \Lambda_{i} \varepsilon S \Lambda_{j} \varepsilon U
\end{gathered}
$$

Sea Porc $=$ como el porcentaje de usuarios que se requiere atender dentro de la región.

Sea $Z_{i}=$ una variable que indica si el AP i se encuentra activo.

Sea $Y_{j}=$ una variable que indica que el usuario $j$ se conecta a un AP.

Sea $X_{i j}=$ una variable que indica que el usuario $\mathrm{j}$ se conecta al AP i.

Tendremos un seudocódigo básico para nuestro algoritmo, Fig. 1.

Fig. 1. PSEUDOCÓDIGO DE AP. COBERTURA

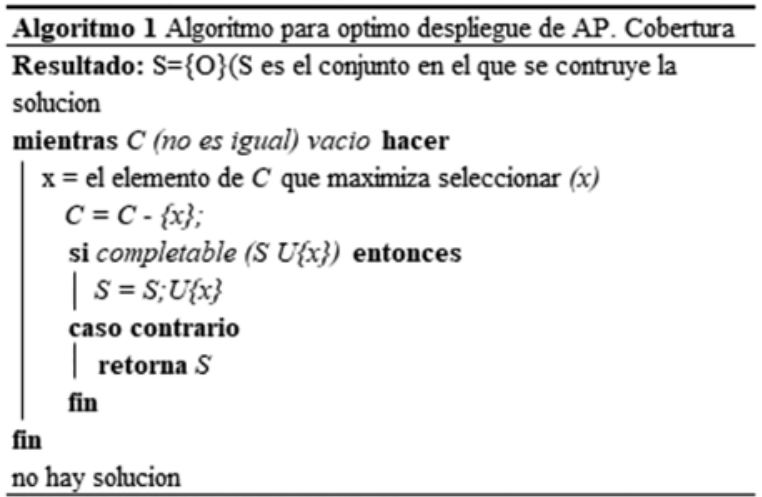

Fuente: Los autores.

\subsection{Cobertura de comunicaciones alámbricas}

Para el estudio del problema por plantear, analizaremos dos algoritmos diferentes en un programa de Matlab, el cual nos indica las diferencias entre el algoritmo de Prim y Kruskal.

Tendremos una tabla de variables:

TABLA II

VARIABLES- KRUSKAL

\begin{tabular}{|c|c|c|}
\hline Variable de decisión & Simbología & Interpretación \\
\hline Conjunto de nodos & $\mathrm{U}$ & Numero de vértices \\
\hline Conjunto de arcos & $\mathrm{V}$ & Numero de lados \\
\hline Grafo & $\mathrm{G}$ & Análisis del gráfico \\
\hline AP & $\mathrm{M}$ & $\begin{array}{c}\text { Número de AP } \\
\text { posibles }\end{array}$ \\
\hline Usuarios & $\mathrm{N}$ & $\begin{array}{c}\text { Número de usua- } \\
\text { rios posibles }\end{array}$ \\
\hline
\end{tabular}

Fuente: Ios autores

En la Tabla II podemos observar cada elemento utilizado en el seudocódigo, a continuación tendremos un seudocódigo básico para nuestro algoritmo, Fig. 2.

Fig. 2. PSEUDOCÓDIGO DE KRUSKAL - PRIM

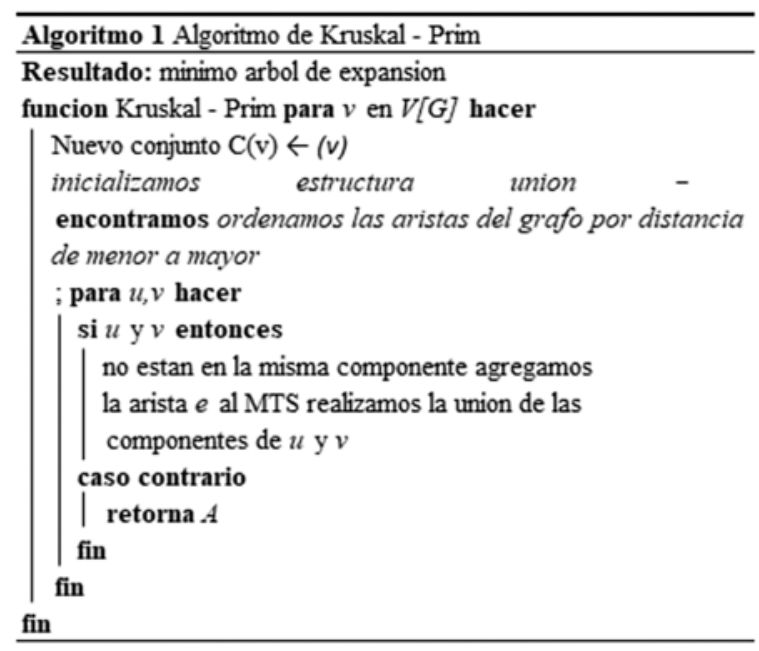

Fuente: Los autores.

\section{ANÁLISIS DE RESULTADOS}

\subsection{Cobertura de comunicaciones inalámbricas}

Para comprobar la cobertura veremos el número máximo de usuarios ubicados en el sector. El algoritmo considera dos variables, que son los valores 
de ubicación de usuarios en el plano georreferenciado.

Para el análisis se quiere determinar la cantidad de posibles ubicaciones en el área seleccionada. Para lo cual se considera los valores de ubicación de los Access Point en el plano georreferenciado. En la simulación también consideraremos el radio de cobertura de cada Access Point que se encuentra establecido por el usuario, coloca la ubicación de cada usuario en el plano, establece la conexión de cada usuario al Access point más cercano de acuerdo con el radio de cobertura. Nos presenta una simulación gráfica con optimización establecida del número mínimo de Access point dependiendo de su porcentaje y radio de cobertura.

El método LPsolve nos presenta una simulación gráfica sin optimización de la conexión de todos los usuarios con los Access point de acuerdo con su radio de cobertura, creando un archivo adicional que nos permite conectar directo con el LP solve. En el LP solve se realiza el resultado de todas las ecuaciones generadas por Matlab para definir los puntos mínimos de Access Point.

En la Fig. 3, Observamos la optimización de las Estaciones Base (4), con una cobertura del 70\%, la cual es la mínima en aplicar, notamos que 11 usuarios quedan fuera del radio de cobertura de la Estación Base.

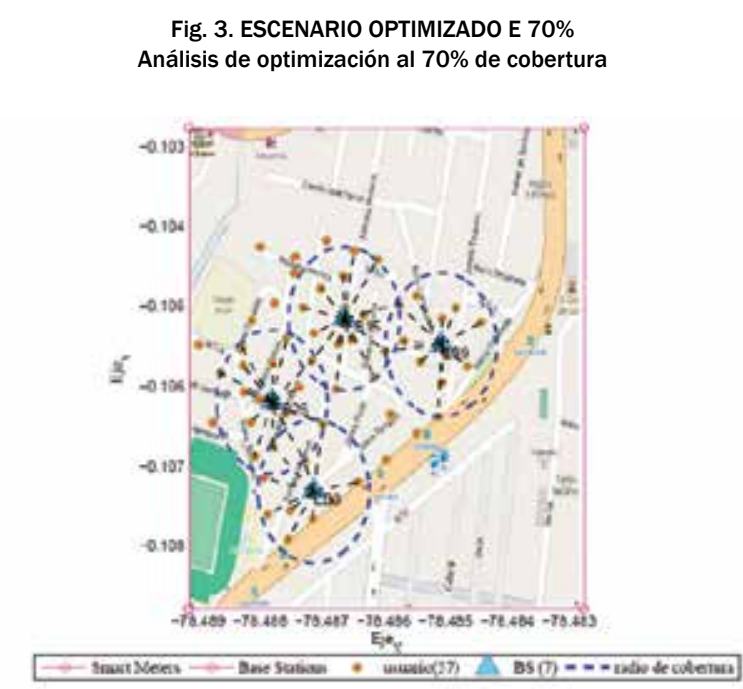

Fuente: Los autores.
En la Fig. 4, Observamos la optimización de las Estaciones base (7), con una cobertura del 100\% a todos los usuarios. En las figuras se observa el análisis de optimización del esquema georreferenciado, los triángulos celestes son las ubicaciones de Estaciones base (7), los círculos tomates representan a las ubicaciones de usuarios (57), las líneas entre cortadas son el radio de cobertura de cada Estación base (15m), las líneas continuas de color café representan a cual Estación Base está conectado cada usuario.

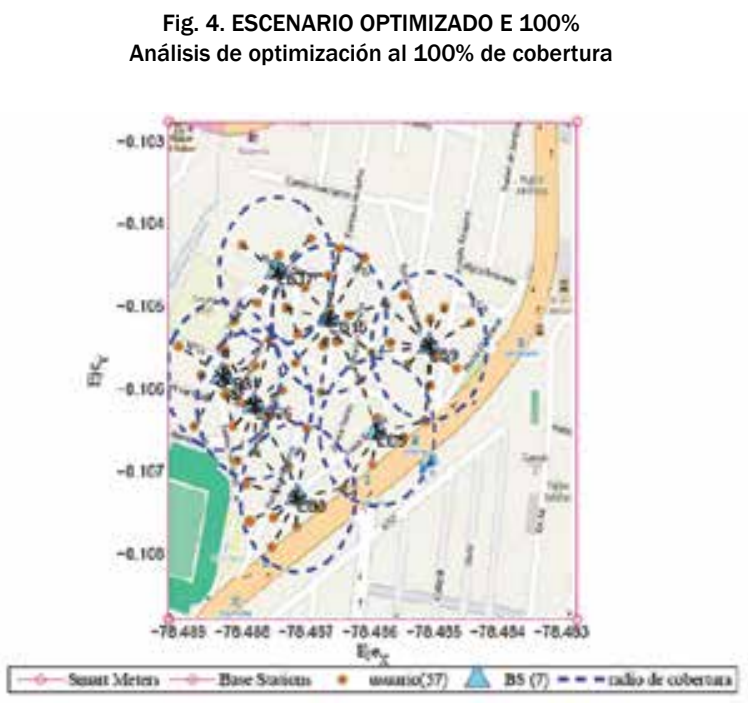

Fuente: Los autores.

\subsection{Cobertura de comunicaciones alámbricas}

A continuación se presenta los resultados obtenidos del uso de los algoritmos de optimización "kruskal" y "Prim", estos algoritmos nos permiten realizar el trazado del árbol de expansión mínima en el software Matlab. La interconexión entre estaciones base es necesario realizarla mediante fibra óptica; la fibra óptica por razones contractivas no se puede llevar a través de terrenos o mediante red aérea, por lo tanto, la manera más óptima para realizar el cableado en las áreas urbanas, para este ejercicio, consideramos, es la optimización de una red subterránea. También para nuestro resultado tenemos la ayuda de LP solve para la optimización de nuestros puntos escogidos.

Así analizaremos los resultados obtenidos y las posibles opciones de colocación, dándonos los costos de metraje por cada método. 
Fig. 5. ALGORITMO PRIM



Fuente: Los autores.

La optimización en el programa Prim, nos ayuda a encontrar un grupo subordinado de aristas que forman un árbol dándonos las conexiones de menor distancia y así tener un menor costo en metraje, en este algoritmo tenemos una conexión adecuada dándonos un costo de metraje de 16.273 dólares, Fig. 5.

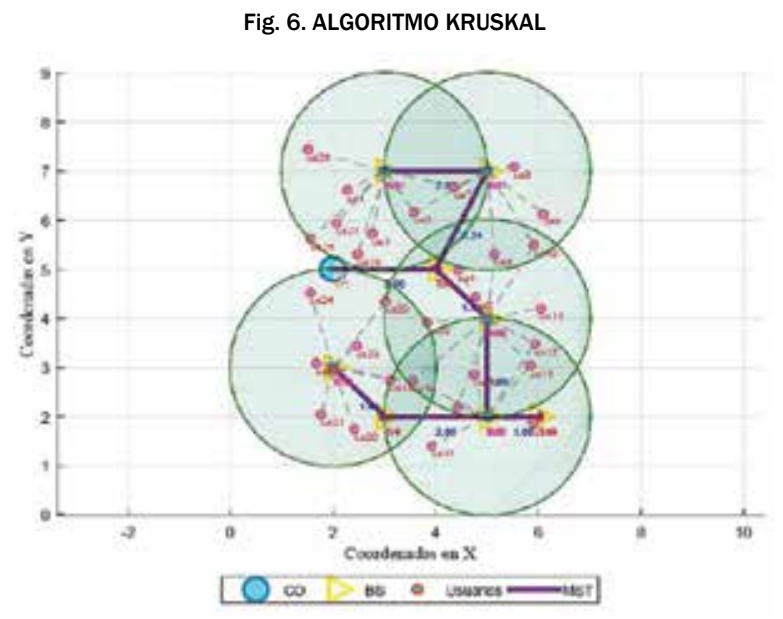

Fuente: Los autores.

La optimización en el programa kruskal es obtener un subconjunto de vértices, teniendo puntos mínimos de selección, con un objetivo de tener menor costo de metraje, Fig. 6. Por el método de Kruskal podemos ver que el costo de metraje es 14.064 dólares.

Así podemos ver que este caso de análisis, nuestro algoritmo de Kruskal nos da un mejor resul- tado de costos, todo depende de su optimización en estos algoritmos, así que su resultado puede variar constantemente.

Esta es una similitud donde los dos documentos comparan el dimensionamiento de la red y la minimización del número de puntos, para la colocación en sitios aleatorios especificando un espacio originario, basándose en la capacidad de cobertura y el radio de alcance del dispositivo. La diferencia que podemos presentar entre estos dos algoritmos es que Kruskal me dará un camino optimo pero también me ayudara a reducir los costos de metraje en cambio el algoritmo Prim me da igualmente el camino optimo pero su metraje siempre será un costo superior, con la utilización de estos algoritmos visualizamos gráficos tanto como: los puntos de acceso, usuarios y el camino óptimo de la red [18].

En un planteamiento óptimo de dimensionamiento para una red, utilizamos muchos puntos de datos para así permitir la transportación de búsqueda, pero con el UDAP podemos reducir las restricciones a lo mismo que estamos realizando en nuestro trabajo, teniendo una minimización de costos. En otros análisis encontramos que buscan la eficiencia y confiabilidad para el consumo de energía. Mediante métodos heurísticos nos permitirá también minimizar costos, teniendo en cuenta la distancia y cobertura de la red y así determinar las ubicaciones óptimas de nuestras estaciones clave [19]-[24].

\section{CONCLUSIONES}

Para la optimización de puntos posibles de conexión de las estaciones bases, se toma datos importantes como el radio cobertura que deseamos, su porcentaje de cobertura y también dependerá del número de usuarios que tendremos en cada estación base. Al desarrollar la optimización de estos algoritmos presentamos podemos ver que cada uno dará su camino más óptimo, claro que toca tomar en cuenta el costo de metraje que nos puede mostrar.

Al permitir el uso de la red FiWi tendremos una mayor calidad de servicio y una optimización correcta con el usuario. Los algoritmos Prim y Krus- 
kal su diferencia se basa de acuerdo a la forma de juntar sus vértices, también debemos añadir que gracias a la ayuda de LP solve podemos tener una cantidad de puntos posibles y mediante los algoritmos reducir a puntos óptimos para el usuario.

\section{AGRADECIMIENTOS}

La elaboración de este trabajo supone un reto desde un punto de vista intelectual e investigador por lo que agradecemos a todo el equipo investigativo que colaboraron durante la realización de este documento, en especial al MSc. Esteban Mauricio Inga Ortega, director de la carrera de Electricidad Campus Sur-Kennedy de la Universidad Politécnica Salesiana Ecuador.

\section{REFERENCIAS}

[1] A. Peralta, E. Inga, and R. Hincapié, "FiWi Network Planning for Smart Metering Based on Multistage Stochastic Programming," IEEE Lat. Am. Trans., vol. 13, no. 12, pp. 3838-3843, 2015.

[2] P. Sarigiannidis and C. Verikoukis, "A Metaheuristic Bandwidth Allocation Scheme for FiWi Networks Using Ant Colony Optimization," Commun. Veh. Technol. Benelux (SCVT), 2015 IEEE Symp., 2015.

[3] S. Singh, R. Srivastava, V. Kumar, and S. Agarwal, "An approximate algorithm for degree constraint minimum spanning tree," 2010 Int. Conf. Comput. Commun. Technol. ICCCT-2010, no. 3, pp. 687-692, 2010.

[4] B. Mondal and T. A. Thomas, "Optimization of Two Layer Macro-Pico Networks Using Lte," 2012, pp. 837841.

[5] D. Chattopadhyay, "Application of general algebraic modeling system to power system optimization," IEEE Trans. Power Syst., vol. 14, no. 1, pp. 15-22, 1999.

[6] Y. Zhou, Z. Luo, and H. Zhuang, "Sensor-assisted coverage self-optimization for wireless local area network," Proc. - 2013 Wirel. Opt. Commun. Conf. WOCC 2013, pp. 444-448, 2013.

[7] S. K. Udgata, K. P. Kumar, and S. L. Sabat, "Swarm intelligence based Resource Allocation Algorithm for cognitive radio network," 2010 First Int. Conf. ParaIlel, Distrib. Grid Comput. (PDGC 2010), pp. 324-329, 2010.
[8] M. F. Munir, "Wireless sensor and sensor-actuator networks: Research trends, protocols, and applications," Incc 2008 leee Int. Netw. Commun. Conf. Proc., p. 6, 2008.

[9] M. Kamenetsky and M. Unbehaun, "Coverage planning for outdoor wireless LAN systems," 2002 Int. Zurich Semin. Broadband Commun. Access - Transm. - Netw. (Cat. No.02TH8599), pp. 1-6, 2002.

[10] E. Amaldi, A. Capone, M. Cesana, and F. Malucelli, “Optimizing WLAN radio coverage," 2004 IEEE Int. Conf. Commun. (IEEE Cat. No.04CH37577), vol. 0, no. c, pp. 180-184, 2004.

[11] E. Amaldi, A. Capone, M. Cesana, F. Malucelli, and F. Palazzo, "WLAN coverage planning: optimization models and algorithms," 2004 IEEE 59th Veh. Technol. Conf., vol. 4, pp. 2219-2223, 2004.

[12] W. Mesh, "Coverage and Capacity of A Wireless Mesh Network," 2005 Int. Conf. Wirel. Networks, Commun. Mob. Comput., pp. 458-463, 2005.

[13] J. Huang, L. Wang, and C. Chang, "QoS Provisioning in Multihop Outdoor Public Access Networks with Asymmetric User Traffic," pp. 5254-5259, 2007.

[14] Q. Dai, G. Shou, Y. Hu, and Z. Guo, "A general model for hybrid fiber-wireless (FiWi) access network virtualization," 2013 IEEE Int. Conf. Commun. Work., pp. 858$862,2013$.

[15] N. Ghazisaidi, M. Maier, and C. M. Assi, "Fiber-wireless (FiWi) access networks: A survey," IEEE Commun. Mag., vol. 47, no. 2, pp. 160-167, 2009.

[16] M. Maier, "FiWi access networks: Future research challenges and moonshot perspectives," 2014 IEEE Int. Conf. Commun. Work., pp. 371-375, 2014.

[17] Y. Yu, C. Ranaweera, C. Lim, E. Wong, L. Guo, and Y. Liu, "Optimization and Deployment of Survivable Fiber- Wireless ( FiWi ) Access Networks with Integrated Small Cell and WiFi," 2015 IEEE Int. Conf. Ubiquitous Wirel. Broadband, no. c, 2015.

[18] C. Ganán, E. Inga, R. Hincapié, C. Ganán, E. Inga, and R. Hincapié, "Óptimo despliegue y enrutamiento de UDAP para infraestructura de medición avanzada basada en el algoritmo MST," Ingeniare. Rev. Chil. Ing., vol. 25, no. 1, pp. 106-115, Jan. 2017. 
[19] D. A. Pérez Cruz, E. M. Inga Ortega, and R. Hincapié, "Optimal sizing of a network for smart metering," IEEE Lat. Am. Trans., vol. 14, no. 5, pp. 2114-2119, May. 2016.

[20] A. Peralta-Sevilla, E. Inga, R. Cumbal, and R. Hincapié, "Optimum deployment of FiWi Networks using wireless sensors based on Universal Data Aggregation Points," in IEEE Colombian Conference on Communication and Computing (IEEE COLCOM 2015), 2015, pp. 1-6.

[21] R. M. Camacho Vera and E. Inga-Ortega, "State of Art, Cognitive Radio for Virtual Network Operator on Advanced Metering Infrastructure," IEEE Lat. Am. Trans., vol. 13, no. 8, pp. 2574-2579, Aug. 2015.
[22] E. Inga-Ortega, A. Peralta-Sevilla, R. C. Hincapié, F. Amaya, and I. Tafur Monroy, "Optimal dimensioning of FiWi networks over advanced metering infrastructure for the smart grid," in 2015 IEEE PES Innovative Smart Grid Technologies Latin America (ISGT LATAM), pp. 3035, 2015.

[23] E. Inga, S. Céspedes, R. Hincapié, and C. A. Cárdenas, "Scalable Route Map for Advanced Metering Infrastructure Based on Optimal Routing of Wireless Heterogeneous Networks," IEEE Wirel. Commun., vol. 24, no. 2, pp. 26-33, Apr. 2017

[24] J. Inga, E. Inga, A. Ortega, R. Hincapié, and C. Gómez, "Optimal Planning for Deployment of FiWi Networks based on Hybrid Heuristic Process," IEEE Lat. Am. Trans., vol. 15, no. 9, pp. 1684-1690, 2017. 